\title{
Intermediate Layers responses to Geomagnetic Activity During the 2009 Deep Solar Minimum Over the Brazilian Low Latitude Sector
}

\author{
Ângela M. Santos ${ }^{1}$, Christiano G. M. Brum ${ }^{2}$, Inez S. Batista ${ }^{1}$, José H. A. Sobral ${ }^{1}$, Mangalathayil A. Abdu ${ }^{1}$, \\ 5 and Jonas R. Souza ${ }^{1}$
}

1National Institute for Space Research, São José dos Campos, Brazil.

2Arecibo Observatory, University of Central Florida, Arecibo, Puerto Rico.

Correspondence to: Ângela Santos (angelamacsantos@gmail.com; angelasantos_1@yahoo.com.br)

10 Abstract. Intermediate layers (ILs) are regions of enhanced electron density located in the ionospheric valley that extends from the peak altitude of the daytime E-region to the bottom side of the F-region. This work presents the daytime behavior of the ILs parameters (the virtual height - h'IL, and the top frequency - ftIL) over the low latitude region of Cachoeria Paulista (CP, $22.42^{\circ} \mathrm{S} ; 45^{\circ} \mathrm{W}$, I: $\left.-34.4^{\circ}\right)$ for the deepest solar minimum of the last 500 years. In such a unique condition, this research reveals for the first time

15 the ILs' quiet state seasonal behavior as well as its responses to moderate changes in the geomagnetic activity. The main results show that even small variations of geomagnetic activity (quantified by the planetary Kp index) are able to modify the dynamics of the ILs parameters. For the first time, it was observed that during the summer, the h'IL decrease rapidly with the increase of geomagnetic activity mainly in the early morning hours. In the following hours, a smoothed rise of the IL was found in all seasons analyzed. Regarding to frequency, it was observed that after 12:00 LT, there is a tendency of it decreased with the increase of the magnetic disturbances, being this characteristic more intense after 16:00 LT, except in the equinox, when little or no response was found during all the interval analyzed. In addition, it stands out that the annual periodicity of the ftIL was observed while the h'IL presents semiannual component.

\section{Introduction}

The deep solar minimum of the solar cycle $23 / 24$ provides an unprecedented opportunity to understand the variability of Earth's ambient ionosphere. During this period, an unusually inactive state 
of the Sun with only relatively small sunspot-carrying active regions was observed. The solar fluxes (UV, $\mathrm{EUV}$, and X-rays) responsible for the heating of the upper atmosphere and production of the ionosphere and the well-known $10.7 \mathrm{~cm}$ solar radio flux $(\mathrm{F} 10.7 \mathrm{~cm})$ presented very low values when compared to the previous solar cycle (see for example Balan et al., 2012 and Kutiev et al., 2013).

Great efforts have been made to better understand the behavior of the different ionospheric layers over the equatorial and low latitudes sectors during this period that was considered the deepest solar minimum of the last 500 years (Hady, 2013). In this context, the effects caused by geomagnetic activities are highlighted, since the variability of radiation coming from the Sun in this case can be neglected. Liu et al. (2012), for example, discussed the impacts of the high-speed stream in the equatorial ionization anomaly (EIA) development. They showed that the inhibition in the EIA formation was probably due to a westward disturbance dynamo electric field. Santos et al. (2016) investigated the behavior of the equatorial $F$ region zonal and vertical plasma drifts over Jicamarca during the weak geomagnetic storm of June 2008. Based on a realistic low-latitude ionospheric model (SUPIM - Sheffield University Plasmasphere-Ionosphere Model), they showed that the perfect anti-correlation between the vertical and the zonal drifts close to the evening prereversal enhancement of the zonal electric field was driven mainly by a vertical Hall electric field induced by the primary zonal electric field in the presence of an enhanced nighttime E region ionization (see Abdu et al., 1998). Sreeja et al. (2011), in their turn, showed that the daytime E-region westward drift over Trivandrum $\left(8.5^{\circ} \mathrm{N}, 77^{\circ} \mathrm{E}\right.$; dip latitude $\left.\sim 0.5^{\circ} \mathrm{N}\right)$ presented a reduction that was simultaneous with the disappearance of the equatorial sporadic $E$ layer $\left(E_{s q}\right)$ echoes in the ionograms. In this case, it was suggested that an additional overshielding electric field (westward/eastward during the day/night), superposed on the ionosphere during the storm main phase contributed to the observed reduction in the drift.

While the effects of the geomagnetic storms on the $E, F$, and sporadic- $E$ (Es) layers are widely investigated, little information can be found about such effects on those layers located in the ionospheric valley, especially during the deep solar cycle minimum of 2008-2009. These layers, which are known as "intermediate layers (or just $I L s$ )" are defined as a region of enhanced electron density located in the ionospheric valley that extends from the peak altitude of the daytime $E$-region to the bottom side of the 
$55 F$-region. As the behavior of the $I L s$ is little discussed in the literature, and in order for the reader to become familiar with the subject that this paper is going to deal with, we will first present a brief summary of the main characteristics of the ILs over the equatorial and low latitudes sectors over Brazil, and then we will be able to understand how the magnetic activity can affect these layers.

Recently, dos Santos et al. (2019) and Santos et al. (2020; 2021) have studied the essential

60 characteristics of the ILs over the Brazilian sector during the minimum and the maximum solar activity epochs and observed that these layers are predominantly diurnal and present a typical downward movement that can last from minutes to hours. Depending on the height at which the ILs are formed, they can descend and merge with the normal ongoing sporadic - $E(E s)$ layers. The ILs' occurrence over Brazil is high and seems to be dependent on the magnetic inclination angle and independent (or weakly

65 dependent) on the solar activity. Nocturnal ILs also were observed over Brazil. Regarding the shape in which the ILs are seen in the ionograms, it was verified that they presented a curved format similar to the "h" type Es layer, however the ILs with a straight format and spreading base appearance also were observed.

The studies conducted so far on the ILs over Brazil give us some indications that the dynamics of 70 these layers can be influenced by the atmospheric tides, gravity waves, and electric fields. The day-today variability in the average $I L s^{\prime}$ descent velocity also suggests the influence of a periodic perturbation with a periodicity of some days. The velocity values found are compatible with those of the semidiurnal and quarter-diurnal tides. However, the larger descending rate (> $10 \mathrm{~km} / \mathrm{h}$ ) observed over the equatorial region may reveal an additional influence of the gravity waves in $I L$ 's dynamics. Additionally, Santos et

75 al. (2021) reported interesting events in which the ILs presented an upward movement at the same time in which the $F$ layer rises due to the evening prereversal enhancement of the zonal electric field. Such characteristic was observed in most of the cases during a period of high solar activity, between October and April months, however a single case also was observed in 2009. Therefore, it is possible that the more intense zonal electric field in 2003 could have an important contribution to the upward movement of the

80 ILs. Another interesting characteristic observed in ILs is that the ILs could suffer in some way the influence of the prompt penetration electric fields. Dos Santos et al. (2019), for example, showed a case 
in which a daytime $I L$ over the equatorial region of São Luis $\left(2^{\circ} \mathrm{S} ; 44^{\circ} \mathrm{W}\right)$ on October 9,2009 presented a strong upward movement that carried the $I L$ to the base of the $F 2$ layer in $\sim 1.5$ hours. This anomalous rise was probably caused by the joint action of the atmospheric gravity wave propagation and the PPEF.

85 Santos et al. (2021) also reported the ascending ILs, however, during sunset times. As mentioned by the authors, it is possible that the ILs in these cases had been caused by the action of the PRE and in some events by an additional contribution from the prompt penetration electric fields. In all the events, the ILs were located at altitudes higher than or equal to $175 \mathrm{~km}$, except the event of November 10, 2003, when an Es layer located at about $120 \mathrm{~km}$ of altitude presented an abrupt rise reaching $290 \mathrm{~km}$ of altitude in a time interval of $\sim 1.25$ hours. This rapid rise of the Es/IL layers probably was caused by an eastward electric field of $\sim 0.6 \mathrm{mV} / \mathrm{m}$ arising from the PRE and the PPEF (for more details, see Santos et al., 2021).

The focus of this paper is to investigate the geomagnetic activity effects on the intermediate layers over the Brazilian low latitude sector during the deep solar minimum of 2009. As mentioned previously, this epoch is especially suited to develop studies like the one proposed here due to the very low values of the solar decimetric flux $(10.7 \mathrm{~cm})$ that were observed. In this case, the effects caused in the $I L s$ by the variability of radiation coming from the Sun can be neglected, and consider only those caused by geomagnetic variations. The data and methodology used to investigate the possible influence of the geomagnetic storms in the intermediate layers is given in Section 2. The results are presented in Section 3 and finally, in Section 4, the discussion and conclusions.

\section{Data set}

In this paper, the ionospheric sounding data collected by the Digisonde operated over the low latitude site, Cachoeira Paulista (CP, $\left.22.42^{\circ} \mathrm{S} ; 45^{\circ} \mathrm{W}, \mathrm{I}:-34.4^{\circ}\right)$, during the deep solar minimum of 2009 are used to verify the possible dependence of the ILs on the geomagnetic activity. The ILs' virtual height ( $h$ ' $I L$ ) and top frequency $(f t I L)$ are analyzed as a function of the Kp index. The ILs data were processed using the SAO Explorer software (Reisnish, 1986) at each 15 minutes. All the observed ILs were included in the analysis, regardless of their descending or ascending movement.

Before going into detail on the topic that this work proposes, we will first give an overview of the behavior of ILs on the sector of CP. Figure 1 shows the variability of the parameter of frequency and 
height of the ILs (panels $\mathbf{a}$ and $\mathbf{b}$ ), their distribution with the local time (panel $\mathbf{c}$ ), as well as their rate of summer (December solstice), winter (June solstice) and equinoxes, respectively. It can be observed that in general the $I L s$ attain higher frequencies (> $6 \mathrm{MHz}$ ) after 11:00 LT (panel a) (except in some cases), and present a high variability in height during all the analyzed period. The downward movement of the $I L s$ is one of the important characteristic that also can be observed in panel $\mathbf{b}$. As indicated in panel $\mathbf{c}$, the

115 occurrence of the ILs along the day is not continuous, which means that the ILs can appear and disappear many times during the day or simply not occur. Additionally, panel $\mathbf{c}$ also shows that in some periods (especially in winter, with some exceptions), there is a tendency that the ILs be formed a little later (after 08-09 LT). Regarding panel d, it can be seen that the ILs occurrence in the low latitude sector of CP increases significantly in the first hours of the day attaining its maximum at 14:00 LT in summer, 12:00

120 LT in winter, $\sim 10: 30 \mathrm{LT}$ in the equinoxes. In general, the probability of occurrence decay drastically as the nighttime period approaches (Note: the seasons were equally divided, i.e., 121 days around the solstices and 61 days around the equinoxes).

(a)
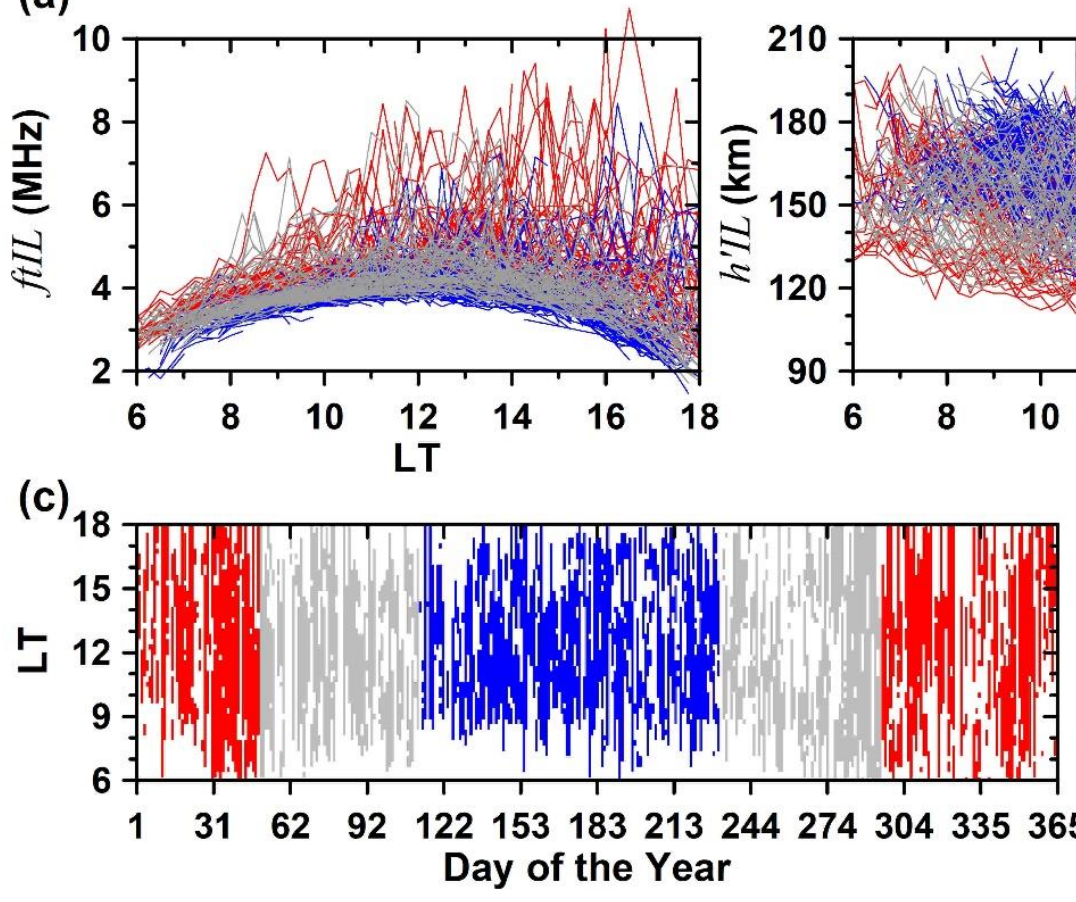

(b)

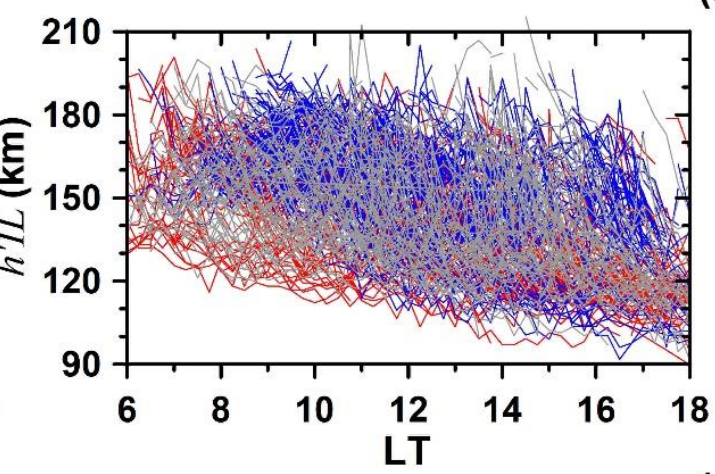

(d)

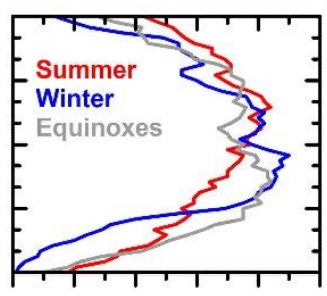

020406080100

Occurrence (days) 
Figure 1: Behavior of the frequency $(f t I L)$ and height ( $h$ 'IL) parameters of the intermediate layers over Cachoeira Paulista during 2009 (panels a and b, respectively) in function of local time; distribution of the ILs occurrence with the local time in function of day of the year for 2009 (c), and; the seasonally occurrence probability of the ILs. In each panel, red, blue and gray are used to represent Summer, Winter and Equinoxes, respectively.

Figure 2 summarizes the geophysical condition of the data distribution according to the solar and geomagnetic activities based on the F10.7P index and $\mathrm{Kp}_{\mathrm{av}}$ index, respectively. The F10.7P (gray line in top left panel) is a combination of the daily decimetric solar flux index (F10.7) and one more term (F10.7A), which corresponds to the average of the 81 previous days, thus $\mathrm{F} 10.7 \mathrm{P}=(\mathrm{F} 10.7 \mathrm{~A}+\mathrm{F} 10.7) / 2$ (given in Solar Flux Units (SFU); $1 \mathrm{SFU}=10^{-22} \mathrm{~W} /\left(\mathrm{m}^{2} \mathrm{~Hz}\right)$ ). F10.7P was chosen because several authors have shown that the ionospheric parameters are better described by this index (Brum et al., 2011 and 2012; Goncharenko et al., 2013). In fact, Brum et al. (2011) and Brum et al. (2012) have shown that the best description of the UV-EUV (based on UV-EUV irradiance data from Pioneer Venus Orbiter (10$150 \mathrm{~nm}$ ) and by the Solar EUV Monitor onboard the Solar Heliospheric Observatory (26-34 nm and 0.1$50 \mathrm{~nm}$ bands)) is given by F10.7P when compared with F10.7. In addition, their works have shown that the UV-EUV emissions tend to increase with F10.7P until a certain threshold (around 175 SFU). However, for low solar activity, the UV-EUV variations with the F10.7P can be well represented by a linear function and this feature is very important for the methodology employed in this work, as seen below. The $\mathrm{Kp}_{\mathrm{av}}$ (gray line in left bottom panel) is the average of the 3 hours data current $\mathrm{Kp}$ value and the previous 3 and 6 hours, that is, $\mathrm{Kp}_{\mathrm{av}}=\left(\mathrm{Kp}_{(\mathrm{ref})}+\mathrm{Kp}_{(\mathrm{ref}-3)}+\mathrm{K} \mathrm{p}_{(\mathrm{ref}-6)}\right) / 3$, which gives the standard behavior of the geomagnetic activity and avoid sharp gradients in the temporal edges of this index (every 3 hours). Then, in the case of $\mathrm{Kp}_{\mathrm{av}}$, different values can be defined per day, since the ILs can occur in different intervals of the day.

The occurrence number in hours of the $\mathrm{Kp}_{\mathrm{av}}$ level in this period is presented on the right bottom panel of Figure 2 (red bars). It is observed that all of the data were acquired during very low to normal 150 geomagnetic activity $\left(\mathrm{Kp}_{\mathrm{av}} \leq 3^{+}\right.$or 3.3) according to the Wrenn et al. (1987) classification. Such distribution is very similar to that found by Terra et al. (2020) when the authors analyzed the MSTID events for the period starting in the middle of 2018 to the end of 2019 (also low solar activity). Note that 
the occurrence of various levels of magnetic activity is well distributed throughout the year (left bottom panel of Figure 2) and this behavior is the optimum condition for the kind of analysis of this work, as will be seen in this report. Regarding the solar activity, the period that encompasses our dataset is the end of solar cycle \#23 and the beginning of solar cycle \#24. A growth of activity and fluctuations of F10.7P along the year is observed, varying from $66.5 \mathrm{SFU}$ to $78.1 \mathrm{SFU}$ (average of 70.1 SFU, top right panel) and an uneven distribution of F10.7P (left upper panel) may be noted. This was a period of very low solar activity in terms of solar flux irradiance (see Emmert et al., 2010) and many works have reported unusual responses of the upper atmosphere/thermosphere under this condition. For instance, during this period, reduction of ionospheric temperatures and densities were detected over several latitudes (Coley et al., 2010; Heelis et al., 2009; Yue et al., 2010; Klenzing et al., 2011; Aponte et al. 2013). Comparing the thermospheric total mass density from the prolonged minimum in solar activity between cycles 23 and 24 with that of the previous solar minima, Emmert et al. (2010) detected a reduction of about 10-30\% compared with the climatologically expected levels. Heelis et al. (2009) and Aponte et al. (2013) reported an unprecedented contraction of the topside ionosphere to altitudes never reported before. 

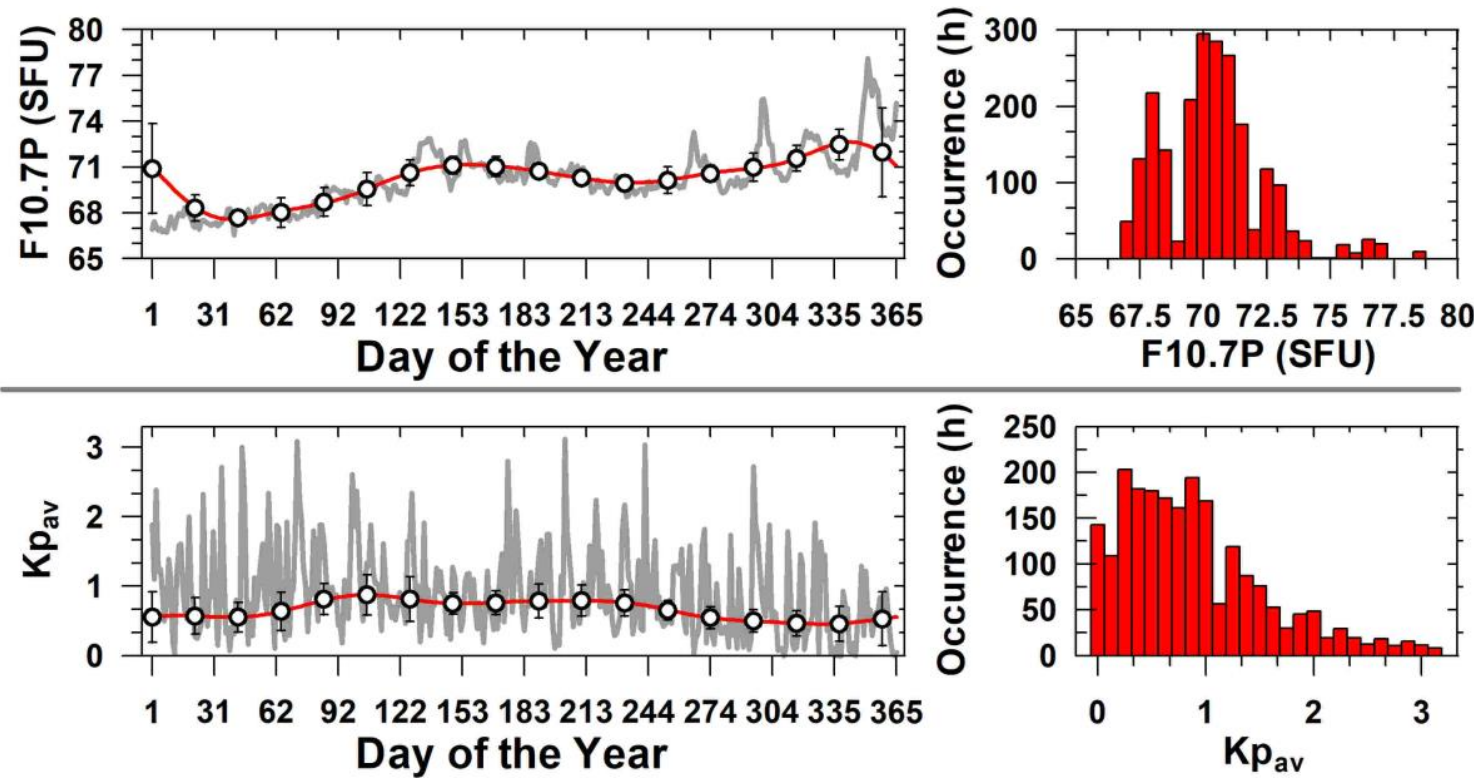

Figure 2: Variability of the solar and geomagnetic activity quantified by the F10.7P and the Kpav indices (upper and bottom block of panels, respectively) for the studied period. Left panels show the geophysical conditions as a function of the day of the year while the right panels their corresponding number of occurrences (in hours) under different geophysical conditions. The dots of the left upper panel represent the 41 days averages and the standard deviation of F10.7P, while dots of the left bottom panel represent the same range of days of the upper panel and its respective standard deviation but for $K \mathrm{p}_{\mathrm{av}}<=2.3$ (geomagnetic condition used to construct the quiet time condition of $h$ 'IL and $f t I L)$ (Note: Left panels show the geophysical conditions as a function of the day of the year (gray line)). The red continuous lines are the reconstruction of these variabilities using Fast Fourier Transform (FFT). The given occurrence in the right column of panels is the number of hours for a given interval of $\mathrm{Kp}_{\mathrm{av}}(0.125)$ and F10.7P $(0.5 \mathrm{SFU})$.

In our study, it has been used the h'IL and ftIL parameters as obtained from the Digisonde observation over Cachoeira Paulista. From these data, an empirical climatological model was developed that accounted for the dependences of these parameters on time and season, under low solar and geomagnetic activities. Determining the variability of $I L s^{\prime}$ parameters in function of time and season make it possible the isolation of any changes related to geomagnetic activity. The first step in our 
methodology was to extract the seasonal quiet time behavior of the $h ' I L$ and ftIL parameters. For this, it was employed the weighted arithmetic mean defined as $x\left(t_{r e f}, d\right)$ represented as (equation 1):

$$
\bar{x}_{\left(t_{r e f}, d_{r e f}\right)}=\frac{\sum_{d_{r e f-20}}^{d_{r e f+20}\left[x_{\left(t_{r e f}, d\right)}\left(\left|d_{r e f}-d\right|\right)\right]}}{\sum_{d_{r e f-20}\left(d_{r e f}-d \mid\right)}^{d_{r e f+20}\left(d_{r}-d\right)}}
$$

190 where $\boldsymbol{x}$ denotes $h$ ' $I L$ or $f t I L$ values under the geomagnetic activity condition below Kpav $\leq 2.3$ for the time reference $t_{r e f}$ and the selected day of the year $(d=D O Y)$. The average value of height and frequency of the ILs was calculated considering 20 days adjacent to the $d_{\text {ref }}$ and 30 minutes around the $t_{\text {ref }}$.

From the quiet time variability of the $h^{\prime} I L$ and $f t I L$ obtained by the 41 days weighted arithmetic mean process a simple model was built using finite Fourier series reconstruction following the procedure by Souza et al. (2010) and Brum et al. (2011), given by

$x V_{(t, d)}=A 0_{(t)}+2 \sum_{m=1}^{4}\left[A m_{(t)} \cos \left(2 \pi m f_{1} d\right)+B m_{(t)} \sin \left(2 \pi m f_{1} d\right)\right]$

where $x V_{(t, d)}$ is the reconstructed variable as a function of time in LT $(t)$ and DOY $(d)(x V$ stands for $h$ ' $I L$ or $f t I L), f_{1}$ is the fundamental frequency of the parameter to be reconstructed $(1 / 365), A 0_{(t)}$ is the annual average of the such parameter for a given $(t)$, and finally, $A m_{(t)}$ and $B m_{(t)}$ are the $m^{\text {th }}$ Fourier coefficients also as a function of time. The terms $A 0_{(t)}, A m_{(t)}$ and $B m_{(t)}$ were incorporated to the model using polynomial fittings in function of time (LT), as shown in Figure 3, for the harmonics $m=1$ (one year), $\mathrm{m}=2$ ( $\sim 6$ months), $\mathrm{m}=3$ ( $\sim 4$ months) and $\mathrm{m}=4$ ( $\sim 3$ months). The upper left ( $\left.h^{\prime} I L\right)$ and right $(f t I L)$ panels show the time dependence of $A 0_{(t)}$ open circles for Fourier coefficients and continuous lines for adjustments). The values of $A m_{(t)}$ and $B m_{(t)}$ are presented in the lower right and left panels by the blue and red curves, respectively, white their best polynomial fitting as well. 

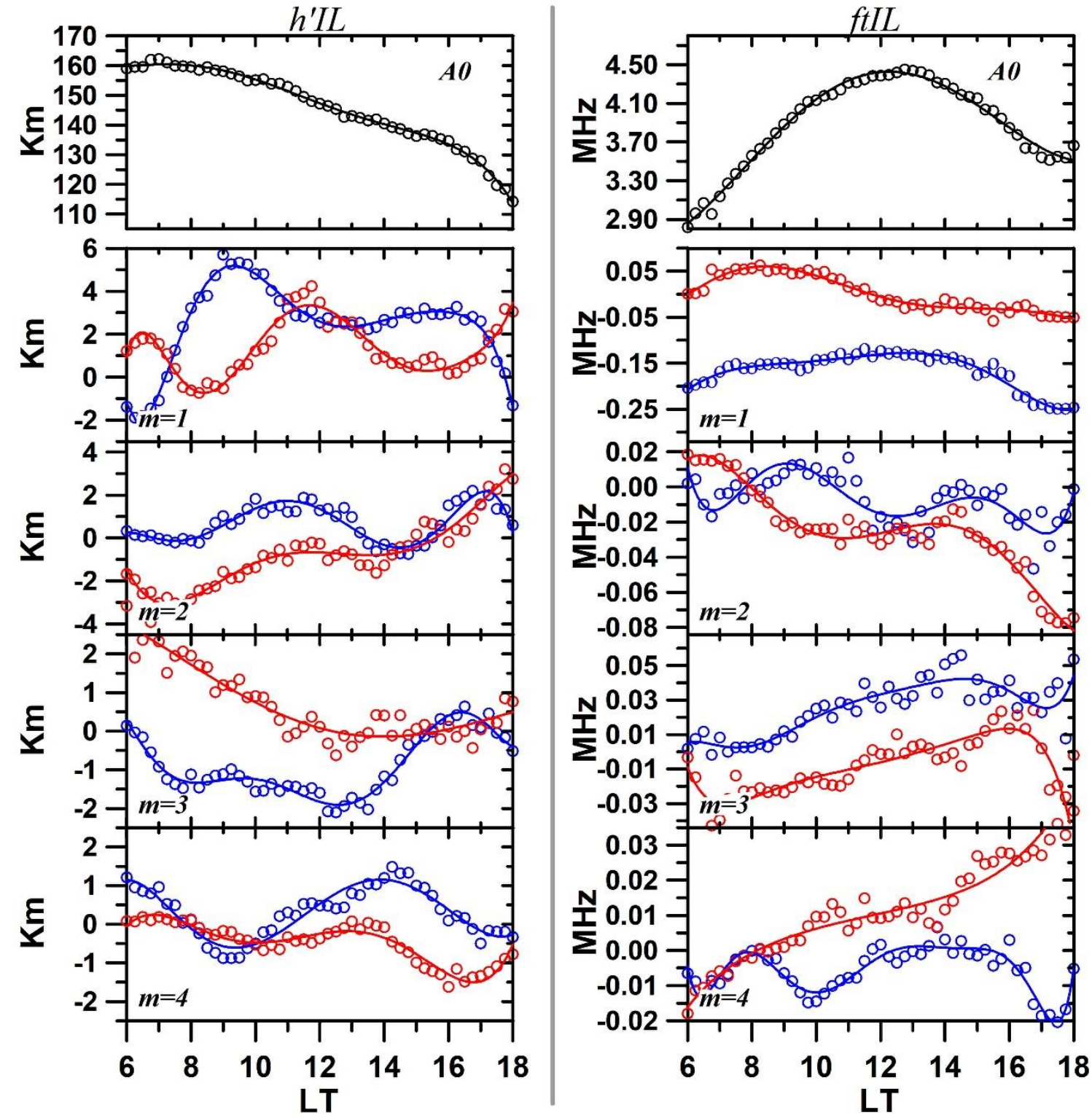

Figure 3: Dependence of the h'IL/ftIL's FFT coefficients as a function of LT (left/right, respectively). The circles are the values obtained by the FFT decomposition, while the continuous lines are the best polynomial approximation (more information in the manuscript body). The colors blue and red are used to represent the coefficients values of $\mathrm{Am}_{(t)}$ and $\mathrm{Bm}_{(t)}$, respectively.

Based on the model output above described, Figure 4 shows the behavior of the $h^{\prime} I L$ and ftIL during the year from 06:00 to 18:00 LT (top and bottom panel, respectively). The right panels show the dispersion diagram between the model and its respective weighted arithmetic mean (under $\mathrm{Kp}_{\mathrm{av}}<=2.3$ ) 
obtained by equation 1 , wherein it is possible to see the good correlation between the observation and the modeled data. The left panels show the dominance of the semi-annual and annual variation of the ILs' virtual height and top frequency, respectively. It is interesting to observe that the upper intermediate layers $(>160 \mathrm{~km})$ are formed as winter approaches in southern hemisphere between $\sim$ 06:00 LT and 11:00 LT, with a maximum in April/May (DOY 92-153) before the local noon. A second maximum is observed from the begging of November to middle of January (DOY 304-15), however, in a more restricted range of time (prior 09:00 LT). After 12:00 LT, the ILs are generally located at altitudes at or below to 150 $\mathrm{km}$. In addition, it is observed that the evolution of the ILs to altitudes below $120 \mathrm{~km}$ was more evident between the months of April and May (DOY 92-153) at the end of the day. The bottom left panel shows an annual variation of the top frequencies, with a maximum at about 12:00-13:00 LT from November to February (DOY 304-62). It can be observed that the upper ILs present lower frequencies when compared to the layers located near $150 \mathrm{~km}$. As the ILs descend, they reach the E region and merge with the existing Es layer, increasing in this way, the top frequency of the layer due to the presence of the metallic ions.
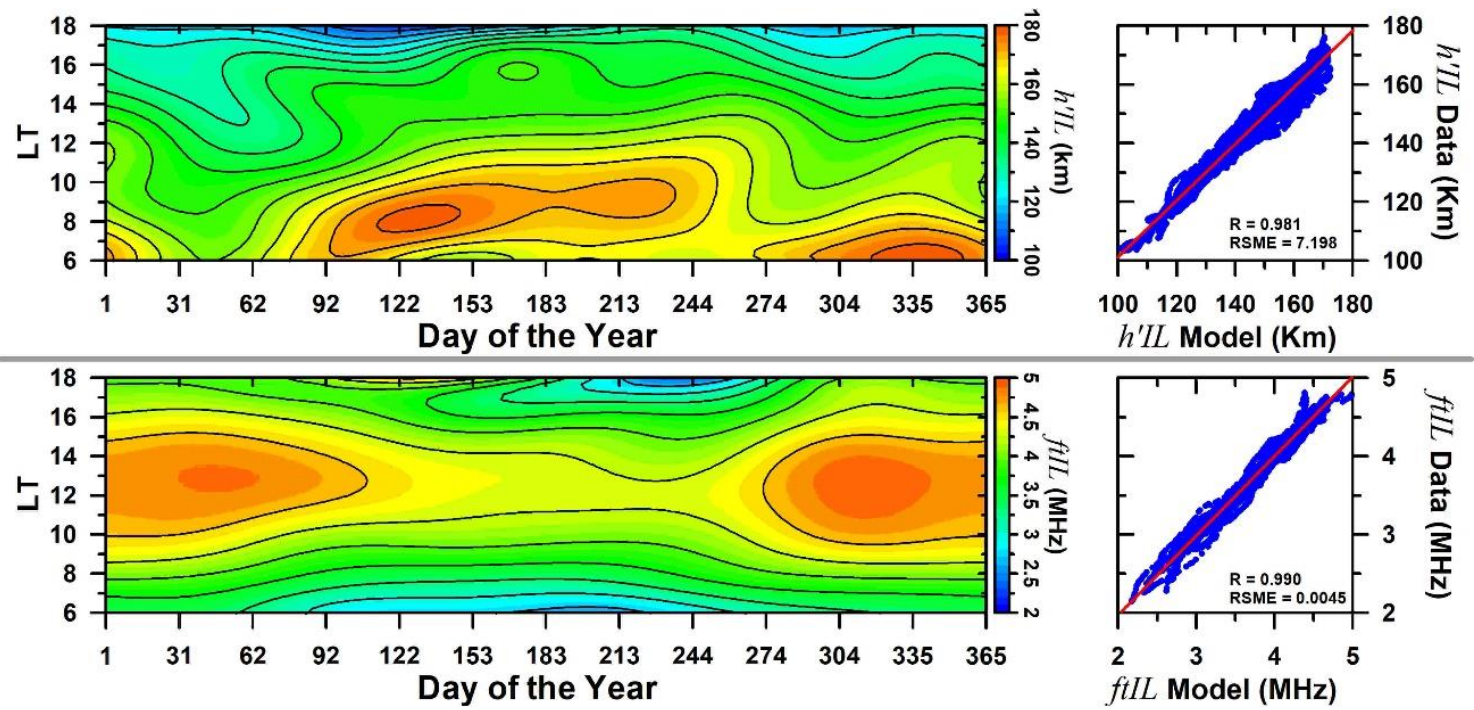

Figure 4: Contour plot of the annual variation of the modeled virtual height (h'IL, left top panel) and top frequency (ftIL, left bottom panel) of the intermediate layers over Cachoeira Paulista. The dispersion diagrams on the right hand-side show the correlation between the weighted arithmetic mean and model results. In each panel on the right, the Correlation Coefficient (R) and Root Mean Square Error (RSME) values are also provided. 
Figure 5 exemplify how the dependence of the different parameters of the intermediate layers in respect to geomagnetic activity was investigated in this work using $\Delta \mathrm{Kp}$ av. The $\Delta \mathrm{Kp}_{\mathrm{av}}$ is the mean of the respective $\mathrm{Kp}_{\mathrm{av}}$ (gray line in the left bottom panel of Figure 2, $\mathrm{Kp}_{\mathrm{av}}=\left(\mathrm{Kp}_{(\mathrm{ref})}+\mathrm{Kp}_{(\text {(ref-3) }}+\mathrm{Kp}_{\text {(ref-6) }) / 3)}\right.$ minus the average of any value below $\mathrm{Kp}_{\mathrm{av}}<=2.3$ in a range of \pm 20 days (this is the geomagnetic condition that the model was developed, the red line shown in the left bottom panel of Figure 2). Noticed: The usage of the residuals minimizes the background quiet time behavior variation along the time (LT and season), enhancing this way the detection of the real contribution or not of the geomagnetic activity on the ILs parameters. The upper panel of Figure 5 shows the whole dataset sorted from the lowest to the highest $\Delta \mathrm{Kp}$ av values and divided into eight sections with the same percentage of samples for each range of $\Delta \mathrm{Kp}$ av (12.5\%, represented by the black vertical lines) for the summer (December solstice) at 17:30 LT \pm 30 minutes. Specifically, for this example, the selected range represents 178 data points, i.e., each $12.5 \%$ displays the behavior of $\sim 22$ individual data samples. This panel also displays the respective F10.7P values (red line) and its respective average and standard deviation (blue open circles) for the same sorted $12.5 \%$ occurrence range of $\Delta \mathrm{Kp}_{\mathrm{av}}$. Note that the $\mathrm{F} 10.7 \mathrm{P}$ mean variation for each range does not vary much which leads us to emphasize that the following variations of $I L S$ are due to geomagnetic activity. The bottom panels show the h'IL and ftIL responses to the geomagnetic activity by the residual average obtained by the difference of the data and the model output presented in Figure 4 in function of $\Delta \mathrm{Kp}_{\mathrm{av}}$.

250 The open circles represent the average values of the height/frequency residuals ( $\Delta h^{\prime} I L$ and $\Delta f t L$, respectively) for the eight different levels of $\Delta \mathrm{Kp}$ av and their respective standard deviations (vertical and horizontal lines). The linear fitting is indicated by the blue lines. The slope (SLP) of the dependence of $h^{\prime} I L$ and $f t I L$ with respect to the geomagnetic activity variation $\left(\mathrm{km} . \Delta \mathrm{Kp}^{-1}\right.$ and $\left.\mathrm{MHz} . \Delta \mathrm{Kp}^{-1}\right)$ and the correlation factor (R) are also shown. In this example, it can be clearly observed that as the geomagnetic 255 activity increase, the height of the intermediate layers also increases. The opposite occurs with the frequency when an increase of the $\mathrm{Kp}$ causes a decrease in this parameter. 

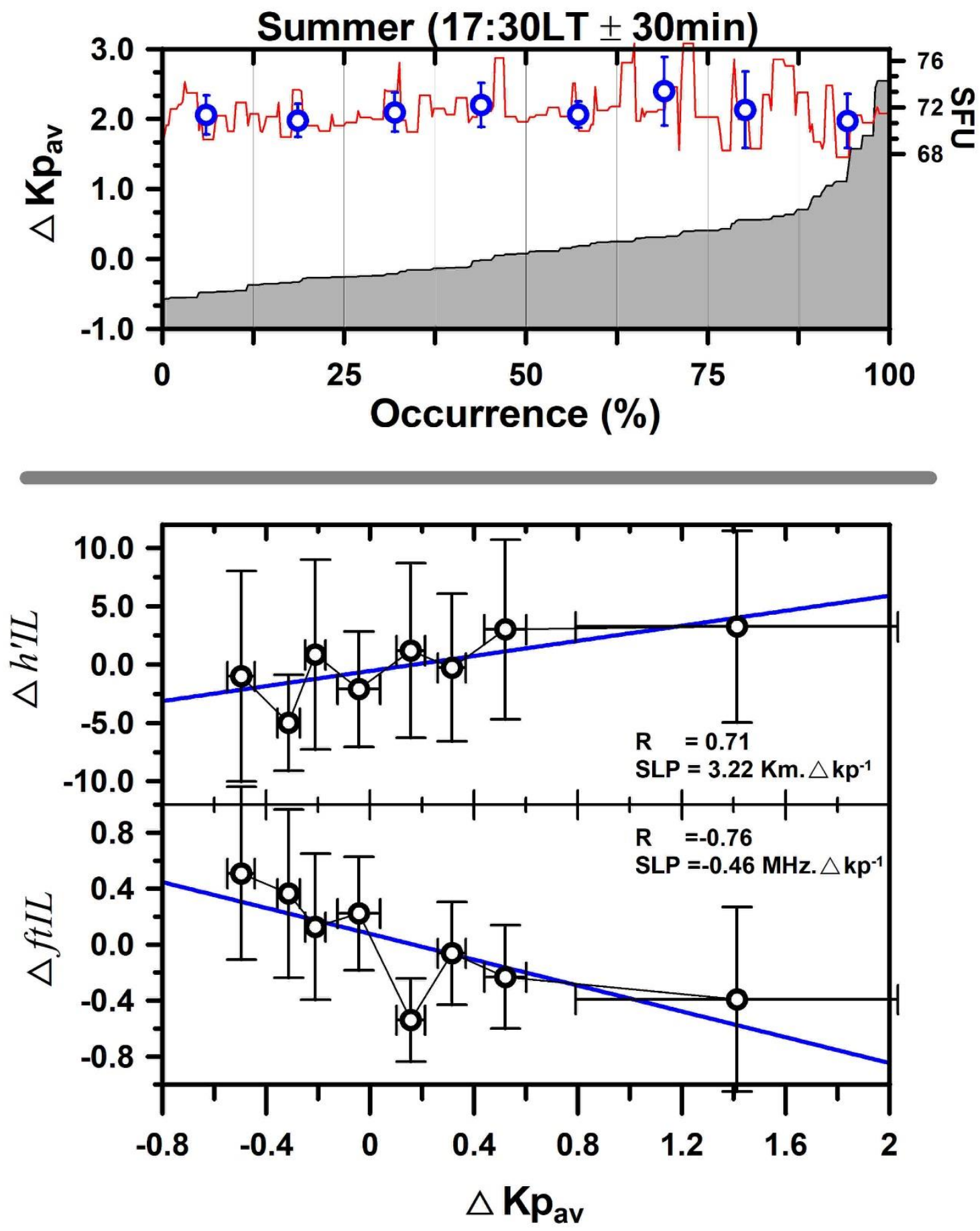

Figure 5: Responses of the intermediate layer to the geomagnetic activity for summer at 17:30 LT \pm 30 minutes. The upper panel shows the $\Delta$ Kpav (see more information in the text) data organized from the lowest to the highest values and divided into eight sections with the same percentage of samples. In addition, the values of F10.7P with respect to 
$\Delta$ Kpav and the average of the F10.7P (blue open circles) for each section are also presented. The bottom panels show the linear regression fitting over the height and frequency residual variability relative to the average $\Delta K p a v$ values.

The same methodology explained in the case of Figure 5 was applied to all the data between 06:00 and 18:00 LT for each season. Due to the lack of data, the night period was excluded from our analysis. Figure 6 shows the ILs' dependence on the geomagnetic activity in terms of height (first two columns from left to right) and frequency (two columns of the left) for different seasons of the year. The data were grouped in summer, equinoxes, and winter. The correlation coefficient $(\mathrm{R})$ of both parameters is also shown at the right column of each block of panels. The variations of the geomagnetic activity presented in this figure were $2.13 \pm 0.28$ (summer), $2.18 \pm 0.24$ (equinoxes) and $1.72 \pm 0.21$ (winter) in Kp index units. It is observed that the higher variability in $I L s$ ' height with geomagnetic activity occurs during the summer period. In this case, the $I L$ goes down fast with the increase of the geomagnetic activity in the beginning of the day. In the following hours, this descent decrease until a moment in which an opposite behavior occurs, that is, a small rise of the $I L$ begins to be observed with the increase of the $\Delta \mathrm{Kp}$ av. Although some fluctuation in the R-value can be observed (mainly during the equinox and winter), there is a tendency that the ILs' height increase with the $\Delta \mathrm{kp}$ variation in all seasons after $\sim 12: 00 \mathrm{LT}$, as can be seen by the positive values of $\mathrm{km} . \Delta K \mathrm{p}^{-1}$. Regarding the behavior of the frequency, it is noticed that in the summer, the $f t I L$ parameter presented a tendency of increase with the geomagnetic activity in the beginning of the day $\left(\sim 0.1 \mathrm{MHz} . \Delta K p^{-1}\right)$, however from $\sim 12: 00 \mathrm{LT}$ on there is a significant decrease in the $f t I L$ with $\Delta \mathrm{Kp}$ av (mainly after 16:00 LT) as can be confirmed by the negative values of the coefficient correlation (R). During the equinox, the general tendency is that the $f t I L$ decrease along the day, and during the winter, little or no response of the top frequency to $\Delta \mathrm{Kp}_{\mathrm{av}}$ variability can be observed prior 16:00 LT and sharp decrease after the referred period similar to what is observed during the summer. 

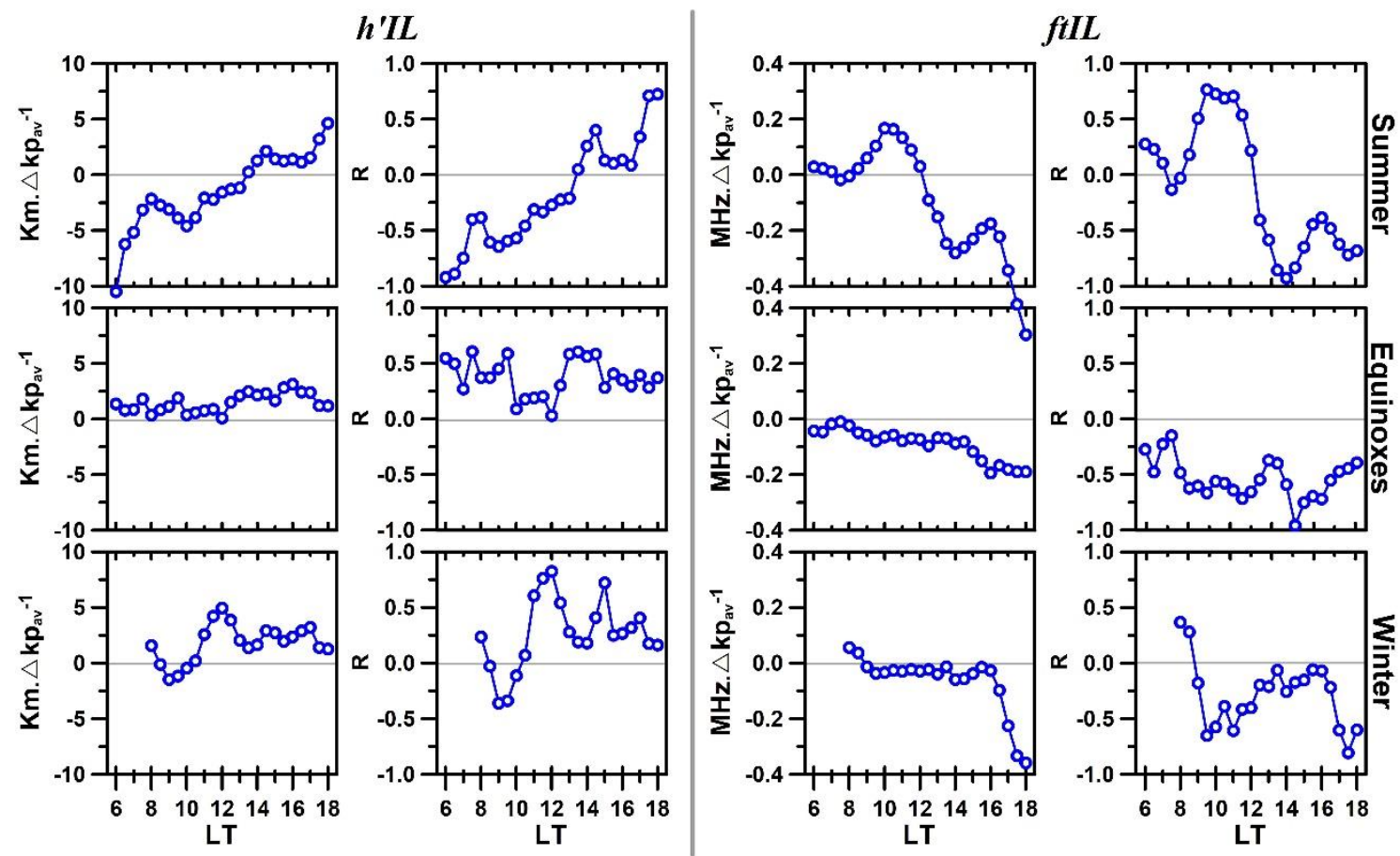

Figure 6: Geomagnetic activity effects on $h$ ' $I L$ and $f t I L$ parameters for different seasons. The first two panels (from left to right) show the linear regression of the $h$ ' $I L$ as a function of the $\Delta \mathrm{Kp}$ av index over the different times of the day and the correlation coefficient $R$. The two right panels indicate the same but for the $f t I L$ parameter.

\section{Discussion and Conclusions.}

It is well known that geomagnetic activity can drastically modify the low-latitude ionospheric dynamics. During the last solar minimum, a unique opportunity was available to investigate such dynamics, since the effects of the solar activity, that dominates the temporal variability of ionospheric properties, could be practically disregarded due to very low solar radiation variation. Using Digisonde data from a Brazilian low latitude station, Cachoeira Paulista, we studied the impacts of the geomagnetic activity in the height and top frequency of the intermediate layers during the deep solar minimum of 2009.

The results summarized in Figure 6 revealed, for the first time, that the most expressive response of the ILs over the low latitude region of Brazil to the geomagnetic activity occurred during the early morning hours ( 06:00 - 08:00 LT) of the summer when the ILs presented a trend of a rapid descent with the increase of the $\mathrm{Kp}_{\mathrm{av}}$ (as indicated by the negative values of $\mathrm{km} . \Delta \mathrm{Kpav}^{-1}$ in $h$ 'IL panels). It is possible 
that this variation in the height of the ILs could have been caused by disturbance electric field having the nature of an over-shielding electric field such as that associated with the northward IMF Bz turning. Such electric fields have westward polarity during daytime and therefore it may be one of the factors responsible for the descending movement of the intermediate layers at this time. As pointed out by Santos et al. (2021), depending on the height at which the ILs are located, the disturbance electric field can affect considerably the vertical displacement of the intermediate layers. They showed that the eastward PPEF can affect partially the rise of the ILs located at higher altitudes (> $170 \mathrm{~km})$ at sunset times. As shown in Figure 4 of the present work, the $I L s$ were located at altitudes very close to this limit (>165 km) between 06:00 LT and 08:00 LT from October to January, reinforcing in this way the idea that a disturbance electric field could be responsible for the downward movement of the ILs.

Another interesting point that needs to be considered is that the movement of the ILs can also be influenced by the regular undisturbed day-to-day variations in the zonal electric field of the ionosphere that is directed to east/west during the daytime/nighttime hours. Therefore, it is possible that in the first 2-3 hours of our analysis period, the descent of the IL could be a result of a competition between the eastward zonal electric field created by the $E$-region dynamo and the disturbance westward electric field arising from the overshielding process. In the following hours, the layer descent movement during the summer and winter slowed down until a moment in which an opposite situation was observed, that is, a 315 small rise of the ILs occurred in all seasonal periods analyzed, as denoted by the positive values of the rate $\mathrm{km} . \Delta \mathrm{Kp}_{\mathrm{av}}{ }^{-1}$ in the first set of panels on the left in Figure 6. As mentioned by Santos et al. (2019), the rise of an $I L$ during daytime can also be a result of the joint action of the of the eastward PPEF (undershielding) and gravity wave propagation. In the case studied by dos Santos et al. (2019), the rise of the $I L$ also was accompanied by a decrease in their top frequency. Wakai (1967) reported that the height 320 of the intermediate layer over Boulder $\left(40^{\circ} \mathrm{N} ; 105^{\circ} \mathrm{W}\right)$ also can be influenced by the magnetic disturbances, however their observation was made during the nighttime.

At the same time in which a decrease in the ILs height is observed, an increase in the ftIL parameter occurs during the summer between 08:00 and 12:00 LT attaining a maximum of 0.2 $\mathrm{MHz} . \Delta \mathrm{Kpav}^{-1}$ at 09:00 LT. It is interesting to observe that before 12:00 LT for example, the rate variation of the $f t I L$ was positive 
indicating that during the downward movement, the ILs' top frequency increased from its initial value. This increase in the frequency is expected since as the layer descends, it can suffer an additional increase of ionization arising from the metallic ions that contribute to the ion density in the ongoing sporadic-E (Es) layers. As the ILs presented a rise after 13:00 LT, the tendency was that the $f t I L$ decrease with the increase of $\Delta \mathrm{Kp}_{\mathrm{av}}$. Note that the after 16:00 LT, this decreased is more accentuated during the summer and winter. Analyzing the incoherent scatter data from the mid-latitude region of Arecibo, Raizada et al. (2017) showed that the integrated electron content ( $E$-region total electron content - ErTEC) between 80 and $150 \mathrm{~km}$ altitude regions presented a maximum variability throughout the night due to geomagnetic activity for both low and high solar activity during equinox periods. Besides that, the authors also verified that the integrated electron content during geomagnetically disturbed/normal conditions and high solar flux periods displays positive changes during summer and equinox, while it is negative in winter. Wakai (1967) reported a study about the maximum electron concentration of the nighttime $E$ layer, the valley above it, and the appearance of the intermediate layer from analysis of the low-frequency ionogram obtained at Boulder on three nights of quiet, moderate, and severe geomagnetic activity. They observed an increase of the ionization in the nighttime valley at times of increased magnetic activity and the appearance of an intermediate layer in $\sim 150-160 \mathrm{~km}$ during periods of moderated activity.

The effects of the magnetic storms on the intermediate layer were studied also by Rodger et al. (1981) using ionosonde data from South Georgia $\left(54^{\circ} \mathrm{S} ; 34^{\circ} \mathrm{W}\right)$. They showed that the rate of the downward movement and the final height of the nocturnal intermediate layer are independent of the season or magnetic activity. Additionally, they observed that the probability of formation of an $I L$ when the minimum virtual height of the $F 2$ layer is above $220 \mathrm{~km}$ is very low, but it can increase during magnetically disturbed periods. As shown by Santos et al. (2020), the ILs' occurrence over Cachoeira Paulista $\left(22.42^{\circ} \mathrm{S} ; 45^{\circ} \mathrm{W}\right.$ ) was very high both in 2009 (a low solar activity year and the same period of this report) and 2003 (a high solar activity year). These results show that in general, the ILs occurrence resulted to be independent of the magnetic disturbances, since the referred two periods of geomagnetic activity are totally different from each other. However, their development/dynamics over Brazilian sector can be affected by disturbed electric fields, as shown by the results here presented and others previous publications (dos Santos et al. 2019 and Santos et al. 2021). 
Although the impacts of the geomagnetic activity on different layers of the ionosphere have been extensively studied, there is a lack of information about what happens in the ionospheric valley region during such conditions, mainly over the low and equatorial latitudes. Using the low-power VHF radar data over the equatorial site of Jicamarca, Chau and Kudeki (2006) showed that the 150-km echoes were not affected by the electric field reversal caused by a magnetic disturbance $(\mathrm{Kp}=5)$. As mentioned by them, a statistical study on the ILs occurrence based on the magnetic activity index Kp did not identify any correlation between magnetic activity and the $150-\mathrm{km}$ echoes. On the other hand, our results show that a small variation in $\mathrm{Kp}_{\mathrm{av}}$ index $(\sim 2.0)$ can affect the $I L s$, especially in the morning period of the summer and late afternoon of all season over the low latitude sector over Brazil. Although the techniques used by us are different from those used by Chau and Kudeki (2006), the contrasting result reveals that the ionospheric valley is a complex region and additional studies need to be performed to understand the physical mechanism governing the generation of the intermediate layers during the occurrence of magnetic disturbances. It is important to emphasize that for the first time it was shown that a small variation in $\mathrm{Kp}_{\mathrm{av}}$ index (by $~ 2.0$ ) was able to impact the dynamics of the intermediate layer over the low latitude region during the period of deep solar minimum. The main results of this work are summarized below:

1. A small variation in the geomagnetic activity during low solar activity can affect both the parameter of height and frequency of the ILs over the low latitude Brazilian sector;

2. During the summer, the height of the ILs suffered a significant decrease with the increase of the magnetic activity magnetic in the first hours of the day. This characteristic was probably caused by an overshielding electric field;

3. During daytime, the smoothed rise of the ILs can be related to the regular day-to-day undisturbed zonal electric field of the ionosphere;

4. After 12:00 LT, there is a tendency that the frequency of the ILs decrease as magnetic disturbances increases, being this characteristic more intense after 16:00 LT, excepted in the equinox, when little or no response was found during all the interval analyzed;

5. A semi-annual and annual variation was observed in parameters of height and top frequency of the ILs, respectively. 
Data availability. The Digisonde data can be downloaded in Zenodo (identified as CAJ2M 2009 in https://doi.org/10.5281/zenodo.3967542).

Author contributions. AMS and CGMB processed the data, performed the analysis and wrote the paper.

ISB, MAA, JHAS, JRS contributed in the interpretation of the data.

Competing interests. The authors declare that they have no conflict of interest.

Special issue statement. From the Sun to the Earth's magnetosphere-ionosphere-thermosphere

Acknowledgments: AMS thanks the financial support from FAPESP (process number: 2015/25357-4) and CNPq (165743/2020-4). The Kp index was obtained from the World Data Center for Geomagnetism, Kyoto (http://wdc.kugi.kyoto-u.ac.jp/index.html) and Solar Radio Flux $(\mathrm{F} 10.7 \mathrm{~cm})$ from the National Oceanic and Atmospheric Administration (NOOA). ISB thanks CNPq grant numbers 306844/2019-2 and 405555/2018-0. One of us (JHAS) had Grant number 303383/2019-4 from the Conselho Nacional de Desenvolvimento Cientifico e Tecnológico (CNPq). J. R. Souza would like to thank the CNPq (grant 395 307181/2018-9) for research productivity sponsorship and the INCT GNSS-NavAer supported by CNPq (465648/2014-2), FAPESP (2017/50115-0) and CAPES (88887.137186/2017-00). The Arecibo Observatory is operated by the University of Central Florida under a cooperative agreement with the National Science Foundation (AST-1744119) and in alliance with Yang Enterprises and Ana G. MéndezUniversidad Metropolitana.

\section{References.}

Balan, N., C. Y. Chen, J. Y. Liu, and G. J. Bailey (2012), Behavior of the low latitude ionospherethermosphere system at long deep solar minimum, Indian J. Radio Space Phys., 41, 89- 97. 
Brum, C. G. M., F. S. Rodrigues, P. T. dos Santos, A. C. Matta, N. Aponte, S. A. Gonzalez, and E. Robles radar and comparison with IRI model predictions for solar cycles 21, 22, and 23, J. Geophys. Res., 116, A03324, doi:10.1029/2010JA015727.

Brum, C. G. M., C. A. Tepley, J. T. Fentzke, E. Robles, P. T. dos Santos, and S. A. Gonzalez (2012), Long-term changes in the thermospheric neutral winds over Arecibo: Climatology based on over three decades of Fabry-Perot observations, J. Geophys. Res., 117, A00H14, doi:10.1029/2011JA016458.

Chau, J. L. and Kudeki, E.: Statistics of 150-km echoes over Jicamarca based on low-power VHF observations, Ann. Geophys., 24, 1305-1310, https://doi.org/10.5194/angeo-24-1305-2006, 2006.

Coley, W. R., R. A. Heelis, M. R. Hairston, G. D. Earle, M. D. Perdue, R. A. Power, L. L. H. B. J. Holt, and C. R. Lippincott (2010), Ion temperature and density relationships measured by CINDI from the 415 C/NOFS spacecraft during solar minimum, J. Geophys. Res., 115, A02313, doi:10.1029/2009JA014665.

Dos Santos, Â. M., Batista, I. S., Abdu, M. A., Sobral, J. H. A., Souza, J. R., \& Brum, C. G. M. (2019). Climatology of intermediate descending layers (or $150 \mathrm{~km}$ echoes) over the equatorial and low-latitude regions of Brazil during the deep solar minimum of 2009. Annales Geophysicae, 37(6), 1005-1024. https://doi.org/10.5194/angeo-37-1005-2019.

Echer, E., B. T. Tsurutani, and W. D. Gonzalez (2012), Extremely low geomagnetic activity during the recent deep solar cycle minimum, Proc. Int. Astron. Union, 7, 200- 209, doi:10.1017/S174392131200484X.

Emmert, J. T., J. L. Lean, and J. M. Picone (2010), Record-low thermospheric density during the 2008 solar minimum, Geophys. Res. Lett., 37, L12102, doi:10.1029/2010GL043671.

Goncharenko, L. P., V.W. Hsu, C. G. M. Brum, S.-R. Zhang, and J. T. Fentzke (2013), Wave signatures in the midlatitude ionosphere during a sudden stratospheric warming of January 2010, J. Geophys. Res.: Space Physics, 118, doi:10.1029/2012JA018251. 
Hady, A. A. (2013), Deep solar minimum and global climate changes. Journal of Advanced Research, V4, I3, P.209-214. doi:10.1016/j.jare.2012.11.001.

Heelis, R. A., W. R. Coley, A. G. Burrell, M. R. Hairston, G. D. Earle, M. D. Perdue, R. A. Power, L. L. H. B. J. Holt, and C. R. Lippincott (2009), Behavior of the $\mathrm{O}+/ \mathrm{H}+$ transition height during the extreme solar minimum of 2008, Geophys. Res. Lett., 36, L00C03, doi:10.1029/2009GL038652.

Klenzing, J., F. A. Simoes, S. Ivanov, R. A. Heelis, D. Bilitza, R. F. Pfaff, and D. Rowland (2011),

Topside equatorial ionospheric density and composition during and after extreme solar minimum, J. Geophys. Res., 116, A12330, doi:10.1029/2011JA017213.

Kutiev I, Tsagouri I, Perrone L, Pancheva D, Mukhtarov P, Mikhailov A, Lastovicka J, Jakowski N, Buresova D, Blanch E, Andonov B, Altadill D, Magdaleno S, Parisi M \& Miquel Torta J (2013), Solar activity impact on the Earth's upper atmosphere. J.Space Weather Space Clim., 3, A06, doi:10.1051/swsc/2013028.

Liu, J., L. Liu, B. Zhao, Y. Wei, L. Hu, and B. Xiong (2012), High-speed stream impacts on the equatorial ionization anomaly region during the deep solar minimum year 2008, J. Geophys. Res., 117, A10304,

Raizada, S.; Brum, C.G.M.; D. Mathews, J.D; Gonzalez, C; Franco, E. (2017). Characteristics of nighttime E-region over Arecibo: Dependence on solar flux and geomagnetic variations, Advances in Space Research, Volume 61, Issue 7, Pages 1850-1857, https://doi.org/10.1016/j.asr.2017.07.006.

Reinisch, B. W.: New techniques in ground-based ionospheric sounding and studies, Radio Sci., 21, 331341, 1986.

Rodger, A. S., Fitzgerald, P. H., and Broom, S. M.: The nocturnal intermediate layer over South Georgia, J. Atmos. Terr. Phys., 43,1043-1050, 1981.

Santos, A. M., M. A. Abdu, J. R. Souza, J. H. A. Sobral, and I. S. Batista (2016), Disturbance zonal and vertical plasma drifts in the Peruvian sector during solar minimum phases, J. Geophys. Res. Space Physics, 121, doi:10.1002/2015JA022146.

Santos, A. M., Batista, I. S., Sobral, J. H. A., Brum, C. G. M., Abdu, M. A., \& Souza, J. R. (2020). Some differences in the dynamics of the intermediate descending layers observed during periods of maximum 
and minimum solar flux. Journal of Geophysical Research: Space Physics, 125, e2019JA027682. https://doi.org/10.1029/2019JA027682.

Santos, Â. M., Batista, I. S., Brum, C. G. M., Sobral, J. H. A., Abdu, M. A., Souza, J. R., \&. (2021). F region electric field effects on the intermediate layer dynamics during the evening prereversal enhancement at equatorial region over Brazil. Journal of Geophysical Research: Space Physics.

Scherliess, L., and B. G. Fejer (1997), Storm time dependence of equatorial disturbance dynamo zonal electric fields, J. Geophys. Res., 102, 24,037-24,046, doi:10.1029/97JA02165.

Souza, J.R., Brum, C.G.M., Abdu, M.A., Batista, I.S., Asevedo, W.D., Bailey, G.J., Bittencourt, J.A. (2010), Parameterized Regional Ionospheric Model and a comparison of its results with experimental data and IRI representations, Advances in Space Research, 46, 1032-1038, https://doi.org/10.1016/j.asr.2009.11.025.

Sreeja, V., T. K. Pant, L. Jose, and S. Ravindran (2011), Westward electric field penetration to the dayside equatorial ionosphere during the main phase of the geomagnetic storm on 22 July 2009, J. Geophys. Res., 116, A03303, doi:10.1029/2010JA016013.

Terra, P., Vargas, F., Brum, C. G. M., \& Miller, E. S. (2020). Geomagnetic and solar dependency of MSTIDs occurrence rate: A climatology based on airglow observations from the Arecibo Observatory ROF. Journal of Geophysical Research: Space Physics, 125, e2019JA027770. https://doi.org/10.1029/2019JA027770

Zerbo J.L., Amory-Mazaudier C., Ouattara F., 2013. Geomagnetism during solar cycle 23: Characteristics. J. Adv. Res, 4(3), 265-274. doi:10.1016/j.jare.2013.08.010.

Wakai, N. (1967), Quiet and disturbed structure and variations of the nighttime E-region, J. Geophys. Res.,72, 4507-4517.

Wrenn, G., Rodger, A. S., \& Rishbeth, H. (1987). Geomagnetic storms in the Antarctic F-region. I. Diurnal and seasonal patterns for main phase effects. Journal of Atmospheric and Solar-Terrestrial Physics, 49(9), 901-913. https://doi.org/10.1016/0021-9169(87)90004-3 
https://doi.org/10.5194/angeo-2021-52

Preprint. Discussion started: 12 October 2021

(C) Author(s) 2021. CC BY 4.0 License.

(c) (1)

480 Yue, X., W. S. Schreiner, J. Lei, C. Rocken, Y. Kuo, and W. Wan (2010), Climatology of ionospheric upper transition height derived from COSMIC satellites during the solar minimum of 2008, J. Atmos. Terr. Phys., 72, 1270-1274, doi:10.1016/j.jastp.2010.08.018. 